$R M x A C, \mathbf{5 3}, 29-34(2021)$

(C) 2021: Instituto de Astronomía, Universidad Nacional Autónoma de México

https://doi.org/10.22201/ia.14052059p.2021.53.07

\title{
WIND DISTURBANCE CANCELLATION FOR SMALLER ALT-AZIMUTH TELESCOPES
}

\author{
Ali Cem Unal ${ }^{1}$, Gokhan Kararsiz ${ }^{2}$, Cemal Tugrul Yilmaz ${ }^{3}$, Onur Keskin ${ }^{1,4}$, and Cahit Yesilyaprak ${ }^{5}$
}

\section{RESUMEN}

Este estudio tiene como objetivo eliminar amplitudes desconocidas producidas como consecuencia de la perturbación del viento que afecta a las monturas altazimutales en pequeños telescopios. Se ha diseñado un controlador adaptativo para minimizar este efecto. El modelo matemático se basa en la configuración altazimutal con 2 grados de libertad, modelándose la perturbación inducida por el viento como una sinusoide con amplitud, frecuencia y fase desconocidas utilizando un modelo de viento más ráfaga. El controlador tiene como objetivo cancelar el efecto de la perturbación en los ángulos de altitud y azimut del telescopio mientras éste se posiciona o permanece estático en una configuración determinada. La estabilidad asintótica se demuestra con el enfoque de Lyapunov. El estudio numérico se muestra en relación al éxito del controlador propuesto.

\section{ABSTRACT}

This study focuses on eliminating unknown amplitude wind disturbance for 2-DOF alt-azimuth configuration small telescopes. An adaptive controller is designed to overcome wind disturbance as a set and forget system. The mathematical model is derived based on 2-DOF alt-azimuth configuration. The wind disturbance is modeled as a sum of sinusoidal with unknown amplitude, frequency and phase by using Wind-Gust model. The controller aims to cancel the effect of the disturbance on the altitude and azimuth angles of the telescope while positioning or staying static on a dedicated configuration. The asymptotic stability is proven with the Lyapunov approach. The numerical study is illustrated to success of the proposed controller.

Key Words: atmospheric effects — instrumentation: adaptive optics

\section{INTRODUCTION}

The ground telescopes are categorized into three sub-groups; small, moderate and large. Moderate and large telescopes are equipped with advanced control and operational systems. The small telescopes are suitable to be equipped with such systems for economical reasons. It can be said that the small telescopes use primitive control systems. This paper uses this drawback as an advantage and introduces an adaptive controller for that field.

In years, academicians applied many control approaches to solve positioning and tracking problems for antennas and telescopes. There are related pa-

\footnotetext{
${ }^{1}$ Center for Optomechatronics Research and Application (OPAM), Isik University, Mesrutiyet Mahallesı Universite Sokak No 2, 34980, Sile, Istanbul, Turkey (cem.unal@isikun.edu.tr).

${ }^{2}$ Department of Mechanical Engineering, Yildiz Technical University, Istanbul, Turkey (kararsiz@yildiz.edu.tr).

${ }^{3}$ Department of Mechanical Engineering, Bogazici University, Istanbul, Turkey (cemal.yilmaz@boun.edu.tr).

${ }^{4}$ Department of Mechanical Engineering, Isik University, Mesrutiyet Mahallesı Universite Sokak No 2, 34980, Sile, Istanbul, Turkey (onur.keskin@isikun.edu.tr).

${ }^{5}$ Center for Astrophysical Application and Research (ATASAM), Erzurum Ataturk University, Erzurum, Turkey (atasam@atauni.edu.tr).
}

pers with this topic in the literature; general control theories for telescopes (Gawronski \& Wodek 2005), (Gunnels \& Steve 2004) and tracking systems for telescopes (Chang\& Po-Kuang 2008).

Plenty of techniques are applied to small telescopes up to now; $H_{\infty}$ (Schonhoff \& Ulrich 2000), PID control (LI 2009), (Zhang \& Bin 2010) and LQG control (Petit 2014).

There is an academic study about optical tracking with adaptive control for optical ground telescopes (Mei \& Rong 2015). In this paper, Rong Mei, Mou Chen and William W. Guo consider the unknown external effect, actuator saturation and parametric uncertainty, simultaneously. However, their telescope system consists of a degree of freedom. This situation motivate us that there are still openings in the literature of telescope controller design.

In this study, alt-azimuth configuration for small telescopes is considered. The aim is orienting the focal point according to tracking a course or pointing on a desired area. Those information are received from a database which astronomers use. The motors are controlled by an adaptive controller which is designed to eliminate disturbance to ensure better positioning. 


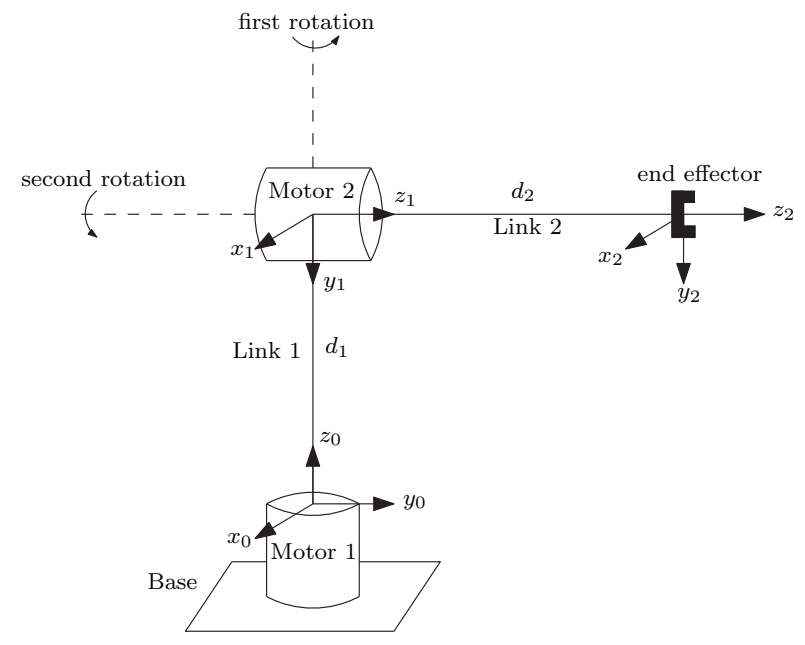

Fig. 1. Coordinate frame definition for modeling motion of the telescope.

Content of this paper: Equation of motion and the telescope model are derived on the second section. The proposed controller is explained on the third section. On the following section, plant assumptions of the system is shown. The unmeasured disturbance is parametrized on the section four. On the fifth section, adaptive controller design is introduced. Then control stability of the system is proved by Lyapunov approach (Johansson 1985) in the following section. The simulation results are given on the seventh section. Lastly, the simulation results and real life application are discussed.

\section{MATHEMATICAL MODEL}

Figure 1 illustrates a small ground based optical telescope with two rotational joints. Denavit Hartenberg representation is used to obtain the mathematical model of the telescope. The detailed explanation of the method can be found in the references (Craig \& John 2005) and (Spong \& Mark 2005).

Inserting the mathematical model of the telescope into Euler-Lagrange method provides the torque equation of the motors. Accelerations are extracted from torque equations. The acceleration equations are linearised around reference. $T_{1}$ and $T_{2}$ are the motor torques, $\dot{\theta}_{1}$ and $\dot{\theta}_{2}$ are represent the motor speeds. $\alpha$ represents the coefficients after simplification of the equations.

$$
\begin{gathered}
\ddot{\theta}_{1}=\alpha_{1} \cdot T_{1}+\alpha_{2} \cdot T_{2}+\alpha_{3} \cdot \dot{\theta}_{1}+\alpha_{4} \cdot \dot{\theta}_{2}+\alpha_{5} \\
\ddot{\theta}_{2}=\alpha_{6} \cdot T_{1}+\alpha_{7} \cdot T_{2}+\alpha_{8} \cdot \dot{\theta}_{1}+\alpha_{9} \cdot \dot{\theta}_{2}+\alpha_{10} .
\end{gathered}
$$

\section{PLANT ASSUMPTIONS}

In this paper, 2-DOF telescope plant under the effect of unknown wind disturbance is considered. It is assumed that all states are available for measurement except the disturbance signal. The aim of the controller is following a reference signal with zero error despite the effect of the disturbance. The following assumptions with regard to plant are considered.

Assumption 1. All states of the system are available for measurement.

Assumption 2. It is assumed that the unknown wind disturbance consists of sum of sinusoidal signals with unknown amplitudes, frequencies and phases.

The bounded disturbance signal $\nu(t)$ is added to the plant (1)-(2) as following

$$
\begin{aligned}
& \ddot{\theta}_{1}=\alpha_{1} \cdot T_{1}+\alpha_{2} \cdot T_{2}+\alpha_{3} \cdot \dot{\theta}_{1}+\alpha_{4} \cdot \dot{\theta}_{2}+\alpha_{5}+\nu \\
& \ddot{\theta}_{2}=\alpha_{6} \cdot T_{1}+\alpha_{7} \cdot T_{2}+\alpha_{8} \cdot \dot{\theta}_{1}+\alpha_{9} \cdot \dot{\theta}_{2}+\alpha_{10}+\nu
\end{aligned}
$$

where $\nu$ represents unknown disturbance. The system dynamics are shown in state-space format as below;

$$
\dot{X}(t)=\left[\begin{array}{c}
\dot{\theta}_{1} \\
\ddot{\theta}_{1} \\
\dot{\theta}_{2} \\
\ddot{\theta}_{2}
\end{array}\right]=A X(t)+B T(t)+D \nu(t)+H \sigma
$$

$A, B, D, H, \sigma$ are known parameters and open forms are given in the appendix. The state $X(t)$ is measured by sensors.

Sinusoidal disturbance is represented as following;

$$
\nu(t)=d+\sum_{i=1}^{q} g_{i} \sin \left(\omega_{i} t+\phi_{i}\right)
$$

where $d$ is a bias term. $g_{i}, \omega_{i}$ and $\phi_{i}$ represent amplitude, frequency and phase respectively. $i \neq j \rightarrow$ $\omega_{i} \neq \omega_{j}, d, g_{i}, \omega_{i}, \phi_{i} \in \mathbb{R}$ for $i=1, \ldots, q$ are assumed as unknown.

\section{DISTURBANCE REPRESENTATION}

The disturbance is represented as a output of an exosystem.

$$
\dot{W}(t)=S W(t), \quad \nu(t)=h^{T} W(t)
$$

$W \in \mathbb{R}^{2 q+16}$ and $S \in \mathbb{R}^{2 q+1 \times 2 q+1}$. Also, $h \in \mathbb{R}^{2 q+1}$ is unique.

The $S$ matrix is depending on the disturbance. The unknown amplitude of the disturbance is related

\footnotetext{
${ }^{6} \mathbb{R}$ represents the set of real numbers and $q$ indicates its dimensions.
} 
to the constant phase and the initial conditions of 7. $d, \omega_{i}, g_{i}$ and $\phi_{i}$ are considered as unknown. Yet, the output vector $h^{T}$ is chosen, the pair $\left(h^{T}, S\right)$ is observable.

It is supposed that $G \in \mathbb{R}^{2 q+1 \times 2 q+1}$ which is a Hurwitz matrix with distinct eigenvalues and the pair $(G, l)$ is controllable. $\left(h^{T}, S\right)$ is observable, $S$ and $G$ have distinct eigenvalues, the solution of this equation is the nonsingular matrix $M$ which $M \in \mathbb{R}^{2 q+1 \times 2 q+1}$ is a solution of the Sylvester Equation.

$$
M S-G M=l h^{T} .
$$

The equation above is invertible. Then, the exosystem has the following after this coordinate transformation $z=M W$;

$$
\begin{aligned}
\dot{z} & =G z+l \nu \\
\nu & =\theta^{T} z \\
\Theta^{T} & =l h^{T} M^{-1} .
\end{aligned}
$$

Lemma 1. The disturbance is parametrized as

$$
\nu=\Theta^{T}(\xi+\delta)
$$

where the filters are designed as following;

$$
\begin{aligned}
& \xi=\eta+N X(t) \\
& \dot{\eta}=G \xi-N(A X+B T+H \sigma)
\end{aligned}
$$

where $N D=l$. Proposed error equation satisfies the following equation;

$$
\dot{\delta}=G \delta
$$

Proof. Proposed error function are defined as following;

$$
\delta=z-\xi
$$

By using (9) and (13)-(14), time derivative of $\delta(15)$ is achieved. In the light of (10), (12) is obtained.

Substituting (12) into (3) and (4), we get the new system equations;

$$
\dot{X}(t)=A X(t)+B T(t)+D\left(\Theta^{T} \xi(t)+\theta^{T} \delta(t)\right) .
$$

Figure 3 shows the time dependent wind model. This wind model is based on Wind-Gust approach (Waslander \& Steven 2009), (Lin 2003).

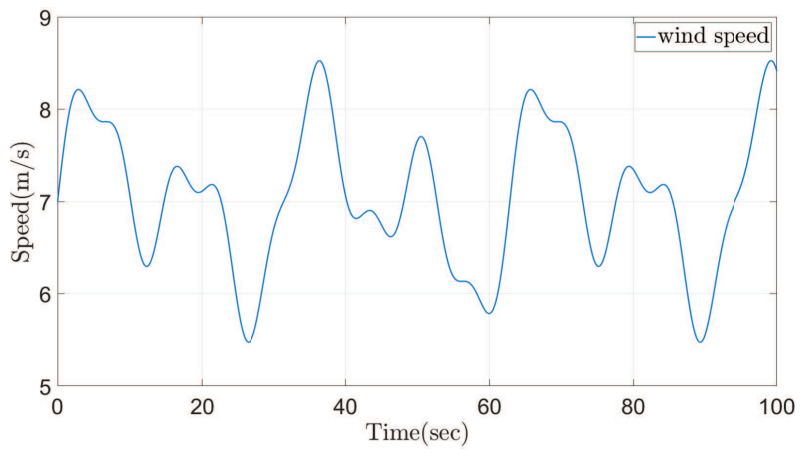

Fig. 2. Wind Model.

\section{CONTROLLER DESIGN}

In this section, an adaptive controller which achieves the reference tracking of the system states is designed. Towards that aim, we take two steps because of the unmatched condition between disturbance and input signal. In the first step, we define the error term, $E_{1}$, for the difference between measured states and reference signals, i.e.

$$
\underbrace{\left[\begin{array}{c}
e_{1} \\
e_{2} \\
e_{3} \\
e_{4}
\end{array}\right]}_{E_{1}}=\underbrace{\left[\begin{array}{c}
\theta_{1} \\
\dot{\theta}_{1} \\
\theta_{2} \\
\dot{\theta}_{2}
\end{array}\right]}_{X}-\underbrace{\left[\begin{array}{c}
\theta_{1 r e f} \\
\dot{\theta}_{1 r e f} \\
\theta_{2 r e f} \\
\dot{\theta}_{2 r e f}
\end{array}\right]}_{X_{\text {ref }}} .
$$

The desired input signal $U(t)$ is feeding to the system directly which rejects the disturbance and drives the error $E(t)$ to zero. The error dynamic of the system is defined as the following;

$$
\dot{E}(t)=(A+B K) E(t)+D\left(\tilde{\Theta}^{T} \xi(t)+\Theta^{T} \delta(t)\right)+H \sigma
$$

where the matrices F, R, Y, M satisfy

$$
\begin{aligned}
& B F=A, \\
& B R=D, \\
& B Y=H, \\
& B M=I .
\end{aligned}
$$

The input signal is designed to satisfy the error dynamics;

$$
T(t)=-F X-R \hat{\theta}^{T} \xi-Y \sigma-C E
$$

where the update law is

$$
\dot{\hat{\theta}}(t)=E^{T}(t) P D \xi(t)
$$




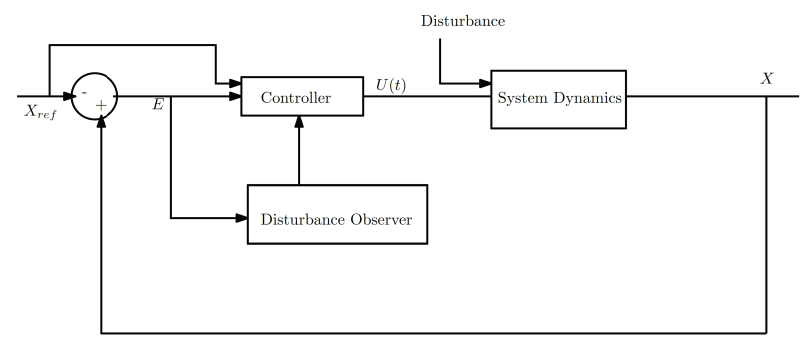

Fig. 3. Logic scheme of the controller.

\section{STABILITY PROOF}

Theorem 1. The closed loop system which is described by the plant (5) forced by an unknown wind disturbance (6) and manipulated by the controller (21). Assumptions $1-3$ hold: the equilibriums of the closed loop systems (19) is stable and $E_{1}(t)$ converges to zero as $t \rightarrow \infty$.

Proof. the following Lyapunov function is proposed to prove the stability of the plant;

$$
V=\frac{1}{2} E_{1}^{T} P E_{1}+\frac{1}{2 \kappa} \tilde{\Theta}^{T} \tilde{\Theta}+\delta^{T} P_{G} \delta
$$

where $P$ and $P_{G}$ are positive definite matrices which are satisfying

$$
\begin{aligned}
(A+B K)^{T} P+P(A+B K) & =-q_{1} I \\
G^{T} P_{G}+P_{G} G & =-q_{2} I
\end{aligned}
$$

with

$$
\begin{aligned}
q_{1}= & \gamma_{1}+\frac{1}{2} \\
q_{2}= & \gamma_{2}+\frac{1}{2} \lambda_{\max }\left(\Theta D^{T} P P D \Theta^{T}\right) \\
& +\frac{1}{2} \lambda_{\max }\left(\Theta D^{T} K^{T} K P D \Theta^{T}\right) \\
& +\frac{1}{2} \lambda_{\max }\left(\Theta D^{T} N^{T} N D \Theta^{T}\right)
\end{aligned}
$$

The parameters $\gamma_{1}, \gamma_{2}$ are any positive constants. Taking time derivative of the Lyapunov function (23) considering (24)-(27) and applying Young's inequality, we get

$$
\dot{V} \leq-\gamma_{1} E_{1}^{T} E_{1}-\gamma_{2} \delta^{T} \delta
$$

From (28), it can be said that $\dot{V}$ is a decreasing function, i.e. $\dot{V}<0$. According to the Lasalle's theorem, $E(t), \tilde{\Theta}(t), \delta(t)$ are bounded and $E(t), \delta(t)$ converge to zero, which satisfies the proof of Theorem 1.

The scheme of the controller can be seen in Figure 3. The disturbance directly effects the system

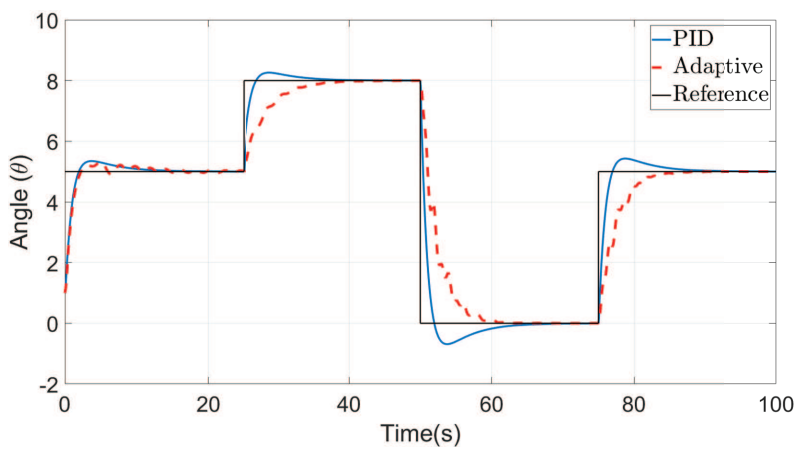

Fig. 4. Telescope positioning output for comparing PID controller and adaptive controller by using reference signal

dynamics. The parameters are updated by feedback method and error $E_{1}$ converges to zero, as it is proven. Error signal is generated by comparing sensor data and reference signal $X_{r e f}$. It is also used by disturbance observer for modifying controller input $U(t)$.

\section{SIMULATION RESULTS}

This part aims to show the performance of the controller. In the simulation the motors of the telescope are working actively under the effect of the wind disturbance whether the telescope following a star or observing and area. The trajectory following is indicated in the simulation as $\theta_{\text {ref }}$. Considering the visual clarity of the simulation, reference points are picked as step points and far away from each other. Gain for the motors are chosen bigger then normal conditions. The Figure 4 illustrates the system behavior. Blue curve shows the position for the PID controller, the red curve shows adaptive controller and black curve shows the reference signal. The wind disturbance is exist from the beginning and $\mathrm{rt}$ changes in time. It can be seen that the adaptive controller has better performance then PID controller.

A PID controller needs tuning with the changing working conditions. Tuning may have done easily, however it can be removed from the system and more practical solution can be achieved in this way. Without tuning step, the controller can be called as a set and forget system and it is build with a adaptive controller.

The Figure 5 is reflecting the torque inputs for the Figure 4. 1st motor controls azimuth motions and 2 nd motor controls altitude motion. The motor 2 generates slightly more torque then the 1st motor because of the gravity. The wind disturbance applied to the plant during the all simulation period. The wind disturbance changes its speed and direction by 


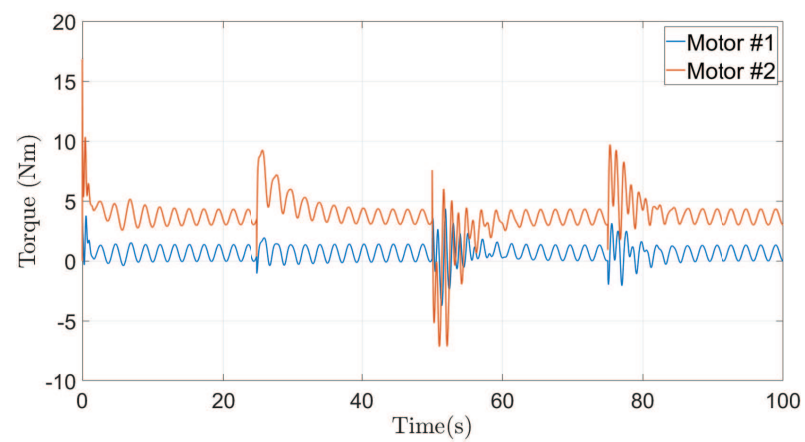

Fig. 5. The torque generated by the motors for following the reference signal on the figure 4.

the time. As a result, the motors are actively canceling disturbance effect even the reference signal is not changing.

The success level of the controller can be seen clearly on the The Figure 6. The comparison of the controllers shows that the adaptive controller is more successful by $\% 20$. This value includes reference tracking and staying still under changing wind disturbance scenarios.

\section{CONCLUSIONS}

In this study, an adaptive backstepping controller is designed to cancel unknown wind disturbance effect for an alt-azimuth configuration optical ground telescope. It provides a set and forget system which doesn't need tuning after installation.

The mathematical description of the telescope is achieved after a couple of steps. First, the position of the focal point according to the ground is found by using forward kinematics approach. The link angles are obtained by applying inverse kinematics method where the configuration information is receiving from star database. After calculating the inertia and velocity matrices, the motor torques are derived by using Lagrangian Approach.

The controller is designed according to the all parameters are known and all states are available for measurement except the wind disturbance signal criteria. An observer is designed to eliminate the unmeasured signal effect. The wind disturbance is modeled as a sinusoidal wave where the frequency, phase and amplitude are unknown. The Lyapunov Function is used to establish the stability of the plant. The simulation illustrates the success of the controller about tracking a reference signal.

The rms between two controllers shows that the adaptive controller is $20 \%$ more successful then PID controller. As it is proposed, adaptive controller doesn't need tuning. This is another contribution to the system besides its better performance.

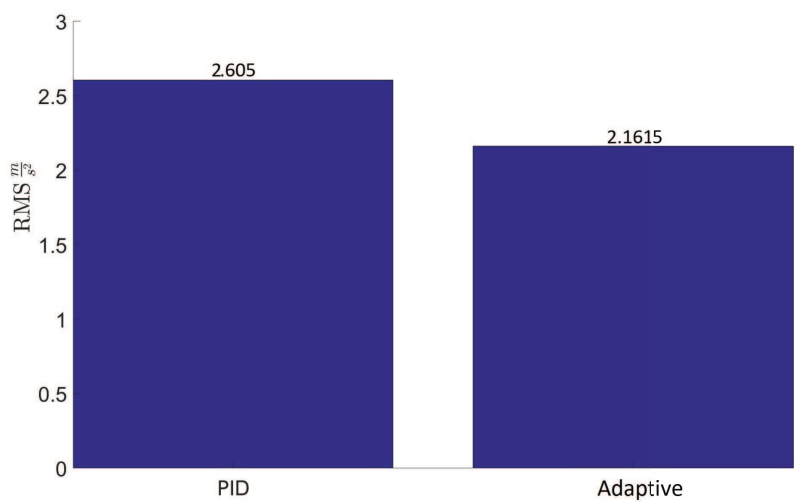

Fig. 6. The comparison of PID and adaptive the controllers in terms of their RMS values.

Open form of the matrices which are given in the equation 5.

$$
\begin{gathered}
A=\left[\begin{array}{cccc}
0 & 1 & 0 & 0 \\
0 & \alpha_{3} & 0 & \alpha_{4} \\
0 & 0 & 0 & 1 \\
0 & \alpha_{8} & 0 & \alpha_{9}
\end{array}\right] \quad B=\left[\begin{array}{cc}
0 & 0 \\
\alpha_{1} & \alpha_{2} \\
0 & 0 \\
\alpha_{8} & \alpha_{7}
\end{array}\right] \\
D=\left[\begin{array}{l}
0 \\
1 \\
0 \\
1
\end{array}\right] \quad H=\left[\begin{array}{ll}
0 & 0 \\
1 & 0 \\
0 & 0 \\
0 & 1
\end{array}\right] \quad \sigma=\left[\begin{array}{c}
\alpha_{5} \\
\alpha_{10}
\end{array}\right]
\end{gathered}
$$

where $\alpha$ and $\mathrm{L}$ are known coefficients according to the individual telescope's link weight and length.

\section{REFERENCES}

Chang, P.-K. \& Lin, J.-M. 2008, Integrating traditional and fuzzy controllers for mobile satellite antenna tracking system design. Proceedings of WSEAS Conference on Advances in Applied Mathematics, Systems, Communications and Computers

Craig, J. J. 2005, Introduction to robotics: mechanics and control, Vol. 3, Pearson Prentice Hall Upper Saddle River

Gawronski, W. \& Souccar, K. 2005, IEEE Antennas and Propagation Magazine, vol 47, no 4, pp 41-49

Gunnels, S., Davison, W. B., Cuerden, B., \& Hertz, E. 2004, SPIE, 5495, 168

Johansson, R. 1985. Global Lyapunov Stability And Exponential Convergence Of Direct Adaptive Control With Recursive Least Squares Identification. Lund.

Li, H.-W. 2009, Servo system of large telescope based on internal model PID control method [J], Optics and Precision Engineering, vol 2

Lin, X. G. 2003, Statistical modelling of severe wind gust, International congress on modelling and simulation, Townsville, vol 2 
Mei, R., Chen, M., \& Guo, W. W. 2015, Optik, 126, 1185

Petit, C., Sauvage, J. -F., Fusco, T., et al. 2014, SPIE, 9148, 0

Schonhoff, U., Klein, A., \& Nordmann, R. 2000, Proceedings of the IEEE Conference and Control, 4, 35763581 Vol. 4

Spong, M. W. 2005, Hutchinson, S. and Vidyasagar, M. M. Robot Modeling and Control
Waslander, S. L. \& Wang, C. 2009, Wind disturbance estimation and rejection for quadrotor position control, AIAA Infotech@ Aerospace Conference and AIAA Unmanned... Unlimited Conference, Seattle, WA

Zhang, B., Li, H.-w., Guo, L.-h., et al. 2010, Application of variable structure PID in velocity control for large telescope, Optics and Precision Engineering, vol 18, no 7, pp 1613-1619 